\title{
La Enseñanza de migración de Datos con el apoyo de la Plataforma Eminus
}

\author{
The Data Migration Teaching with the support of the Eminus Platform
}

CHAVEZ-DIAZ, Leticia †*, HERNANDEZ-VIDAL, Omar, GONZALEZ-COBOS, Claudia y AOYAMA-ARGUMEDO, Esperanza

Universidad Veracruzana, Facultad de Trabajo Social, Calle $12 N^{o} .215$, col. Cazones. Poza Rica, Ver.

ID $1^{\text {er }}$ Autor: Leticia, Chavez-Diaz / ORC ID: 0000-0002-3803-0403, Researcher ID Thomson: I-6708-2018, CVU CONACYT ID: 902209

ID $1^{\text {er }}$ Coautor: Omar, Hernandez-Vidal / ORC ID: 0000-0001-7112-0606, CVU CONACYT ID: 849781

ID $2^{\text {do }}$ Coautor: Claudia, Gonzalez-Cobos / ORC ID: 0000-0002-1461-0198, CVU CONACYT ID: 849217

ID $3^{\text {er }}$ Coautor: Esperanza, Aoyama-Argumedo / ORC ID: 0000-0001-7869-7010, CVU CONACYT ID: 950583

DOI: $10.35429 / J E S C .2019 .7 .3 .31 .39$

Recibido: 08 de Enero, 2019; Aceptado 26 de Marzo, 2019

\section{Resumen}

La investigación denota la importancia que tiene la migración de datos a una plataforma educativa, la cual permite que los docentes y estudiantes participen en un entorno digital a través de las nuevas tecnologías y el manejo de las computadoras, haciendo uso intensivo de las facilidades que proporciona Internet y las tecnologías digitales a través de la plataformas institucionales, por eso es necesario utilizar las herramientas que ofrece la Universidad Veracruzana (UV) con la finalidad de manejar y migrar la información virtual, así mismo incursionar en las diferentes demandas en las carreras que ofrecen actualmente, abordando estos cambios en los nuevos programas de estudio que puedan ser Online, lo que permite actualizar el proceso de Enseñanza Aprendizaje y obtener un cambio en los estudiantes y docentes. El objetivo es manejar las herramientas en modalidad presencial como virtual Implementando el uso de plataformas online. Por lo tanto, esta investigación permite responder cuales son los beneficios de migrar la información, contenidos, foros, tareas, multimedios a las plataformas. Este trabajo será de corte cuantitativo, método descriptivo como eje integrador y colaborador del logro de objetivos que implementa la Universidad.

Enseñanza, Plataforma Eminus, Migración

\begin{abstract}
The research denotes the importance of data migration to an educational platform, which allows teachers and students to participate in a digital environment through new technologies and computer management, making intensive use of the facilities provided Internet and digital technologies through the institutional platforms, so it is necessary to use the tools offered by the Veracruzana University (UV) in order to manage and migrate virtual information, as well as to venture into the different demands in the careers they offer Currently, addressing these changes in the new study programs that may be Online, which allows updating the Learning Teaching process and obtaining a change in students and teachers. The objective is to manage the tools in face-to-face and virtual mode Implementing the use of online platforms. Therefore, this research allows us to answer what are the benefits of migrating information, content, forums, tasks, multimedia to platforms. This work will be quantitative, descriptive method as an integrating and collaborative axis of the achievement of objectives that the University implements.
\end{abstract}

Teaching, Eminus Platform, Migration

Citación: CHAVEZ-DIAZ, Leticia, HERNANDEZ-VIDAL, Omar, GONZALEZ-COBOS, Claudia y AOYAMAARGUMEDO, Esperanza. La Enseñanza de migración de Datos con el apoyo de la Plataforma Eminus. Revista de Ciencias de la Educación. 2019. 3-7: 31-39

\footnotetext{
* Correspondencia del Autor (correo electrónico: Lchavez@uv.mx)

$\dagger$ Investigador contribuyendo como primer autor.
} 


\section{Introducción}

En el presente artículo se trata de mostrar como las plataformas virtuales han influenciado en el aprendizaje virtual teniendo así una gran importancia en las carreras de la Universidad Veracruzana y un impacto significativo en la Educación superior, desarrollando el uso de herramientas de transferencia de conocimientos, que facilita el aprendizaje online, mismo que tiene que ser utilizado por los docentes y estudiantes. transformándose en un recurso optimizador de tiempos reales, capaz de mejorar y potenciar los niveles cognitivos del estudiante por medio de las diferentes aplicaciones que ofrecen las plataformas.

En el siglo XXI el sistema educativo actual se explica como la reorganización del sistema en tres ejes para que los seres humanos desarrollen todo su potencial en la escuela, lo que les permitirá tomar decisiones de una manera integral. El conocimiento vinculado con actividades cotidianas y el uso de nuevas tecnológicas permitirá que el alumno asuma sus experiencias de vida como elementos que le ayudarán en su andar día a día. La diferencias entre el modelo de enseñanza actual y el nuevo, radica en evitar la memorización de datos.

Este nuevo currículo se organiza a partir de tres componentes: Formación académica, que consiste en las asignaturas de observancia nacional; Desarrollo Personal y Social, entre las que se incluyen las habilidades socioemocionales; y Autonomía Curricular, que da a las comunidades escolares la oportunidad de definir una parte del currículo, según sus intereses y necesidades", informó al respecto la Secretaría de Educación Pública a través de comunicado.(2019)

El Sistema Educativo se ha visto inmerso en teorías como el cognitivismo, conductismo y constructivismo, entre otras, que tratan de explicar y apoyar la mejor manera de enseñar y aprender. (Schunk, 2010).

Bajo estas teorías y pretendiendo dar respuesta al ámbito laboral que hoy demandan las empresas en la llamada sociedad del conocimiento, surgen dos conceptos:
El Enfoque por Competencias, que traduce el concepto de "competencias" como un "saber" en el que se utiliza el conocimiento para la resolución de problemas (Torres, 2012) y el concepto Tecnologías de la Información y Comunicación (TIC), definidas como un conjunto de disciplinas científicas, tecnológicas, de ingeniería y de gestión, utilizadas en el manejo y procesamiento de la información, que emplean la computadora como medio (Cabero, 2007).

González (1986) informa que actualmente la demanda social se dirige, tanto a nivel nacional como internacional a la calidad de la educación impartida, donde interesa saber si el egresado de alguna carrera universitaria está capacitado teórica y metodológicamente para enfrentar los retos propios de las funciones laborales que corresponden a su área. Algunos conceptos que aparecen asociados a la calidad del aprendizaje profesional son:
a. La validez y actualidad del conocimiento obtenido.
b. La relación entre la formación y los lugares de trabajo.
c. La práctica en resolver casos tal como sucede en el campo laboral.
d. El autoaprendizaje.
e. Las habilidades cognoscitivas de alto nivel.

De esta manera se retoma algunos puntos esenciales para considerar en la educación superior y que son importantes para su impartición en forma virtual:
a. Diseño y utilización de sistemas inteligentes para enseñanza y aprendizaje con representación avanzada del conocimiento del área que se trate.
b. Trasmisión del saber.
c. Construcción de nuevas estructuras cognitivas.
d. Pedagogía centrada en el autoaprendizaje y poder creativo del estudiante.
e. Creación y utilización de bancos de conocimiento.
f. Trabajo interdisciplinario y multisectorial.


En el modelo de Enseñanza Virtual, el alumnado pasa a ser el principal protagonista y el profesorado transforma sus funciones para proporcionar guía y orientación del método de aprendizaje apropiado

\section{Justificación}

Esta propuesta surge como resultado del uso de la plataforma institucional Eminus de la Universidad Veracruzana, por tres académicos del Área de Formación Básica General AFBG de la Experiencia Educativa de computación básica y una docente de Pedagogía, los cuales evalúan de una u otra forma la calidad de los servicios que se prestan a los estudiantes con el uso de la plataforma virtual, sus contenidos y programas donde docentes y estudiantes utilicen las herramientas para desempeñarse profesionalmente usando el modelo virtual-y semipresencial, donde trabajan de manera integrada y colaborativa, enfocando los cursos de acción de acuerdo a los objetivos, contenidos, materiales de apoyo, actividades, evaluaciones, tiempo y equipo de trabajo; generando resultados que permiten dar continuidad al sistema de Enseñanza virtual (Colunga, 2005),

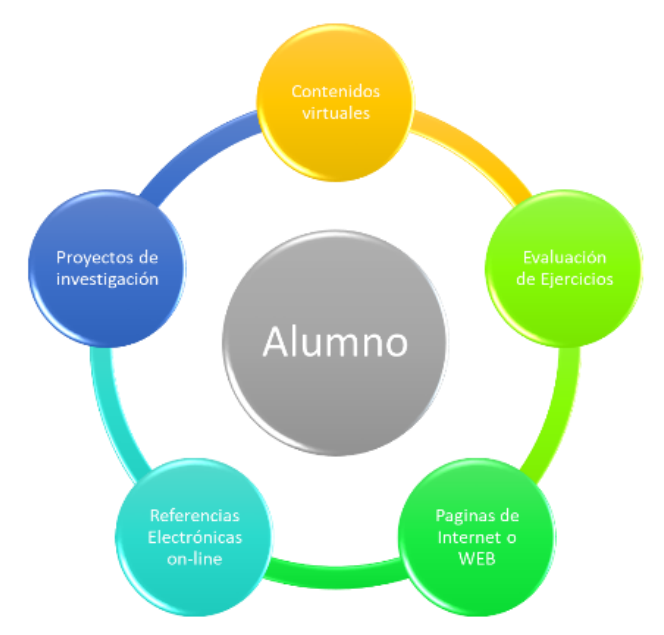

Figura 1 Modelo de enseñanza virtual: Centrado en el alumno o la alumna

Fuente: Elaboración Propia

\section{Planteamiento del Problema}

Es inevitable que estando en el siglo XXI el uso de las plataformas virtuales hayan ganado terreno en la educación y que hoy en día sea de manera virtual e indispensable de aprendizaje y establezca un alto volumen de competencia de mercado escolar con el uso de las TIC, tecnologías de la información y comunicación.
Esto tiene un gran impacto debido a que en muchas universidades ofrecen en tiempo corto, a distancias, online, reduciendo tiempos y costos, de manera personalizada ofreciendo la variedad de información, contenidos y carreras de cualquier tipo $y$ en cualquier lugar facilitándose así el aprendizaje desde tu hogar.

Sofía Victoria (2008) la define a la plataforma virtual como "escenarios educativos diseñados de acuerdo a una metodología de acompañamiento a distancia" o "herramientas basadas en páginas Web para la organización e implementación de cursos en línea o para apoyar actividades educativas presenciales" determinar que las plataformas virtuales educativas son "programas informáticos que llevan integrado diversos recursos de hipertexto y que son configurados por el docente, en función a las necesidades de la formación, para establecer un intercambio de información y opinión con el discente, tanto de manera síncrona como asíncrona".

En la Universidad Veracruzana especialmente en la Facultad de Trabajo Social, se implementó esta herramienta en el proceso enseñanza-aprendizaje, la cual ofrece ambientes de aprendizaje ya diseñados e integrados, cambiando la forma tradicional de desarrollar los cursos en las diferentes experiencias educativas que ofrece en sus planes y programas. Es importante mencionar, que la facultad tiene acceso a una plataforma institucional virtual llamada Eminus, su uso no se ha generalizado en todas las experiencias educativas tampoco cuenta con la aceptación por parte de todos los docentes por diversas razones de manejo.

\section{Objetivos de la investigación}

\section{Objetivo General}

Conocer la situación actual sobre la utilización de la plataforma virtual Eminus por parte de los docentes y estudiantes de la Facultad de Trabajo Social Poza Rica Tuxpan de la Universidad Veracruzana.

\section{Objetivos Específicos}

Determinar el manejo que realizan los docentes y estudiantes en la plataforma Eminus en la Facultad de Trabajo Social Poza Rica. 
Identificar las actividades que han desarrollado los docentes y estudiantes para el manejo de la plataforma Eminus

Describir los beneficios que aporta el uso de la plataforma Eminus en Docentes y estudiantes.

\section{Metodología}

La presente investigación se obtuvo de los trabajos realizados por docentes de las experiencias educativas de computación básica impartiéndose en la Facultad de Trabajo Social Poza Rica Tuxpan en un entorno educativo superior mediante el uso de la plataforma institucional Eminus como apoyo en el proceso de enseñanza aprendizaje virtual presencial, el cual aplica nuevo aprendizaje en entornos virtuales trabajo de los alumnos, modificando el rol de los profesores y la manera de trabajar los contenidos en relación con los sistemas de comunicación y con el diseño y la distribución de la enseñanza- aprendizaje.

De alumno o el alumno es quien "marca" su propio ritmo de trabajo adquiriendo los conocimientos expuestos en los contenidos, haciendo los ejercicios de autoevaluación, consultando y resolviendo dudas con el resto de participantes del curso, realizando las tareas, actividades y el docente es quien va guiándole durante su proceso de aprendizaje. Para ayudar a que todo el alumnado encuentre un ritmo propio, pero adecuado para conseguir el mejor rendimiento, la labor de atención y tutorización del alumnado debe ir estableciendo fechas importantes, foros, contenidos eventos, para marcar un ritmo de trabajo adecuado para el alumnado, como por ejemplo, fechas en tiempo y forma para la realizar de las tareas y subirlas en tiempo y forma a la plataforma Institucional Eminus.

\section{Tipo de investigación:}

La presente investigación se realiza bajo un enfoque cuantitativo, mediante un proceso deductivo que plantea inicialmente analizar el efecto que provoca en el docente y el estudiante sobre la implementación, manejo y uso del aprendizaje mediante la plataforma Eminus en el programa de estudios de la experiencia educativa computación básica.

\section{Marco Teórico}

Un modelo educativo consiste en una recopilación o síntesis de distintas teorías y enfoques pedagógicos que orientan a los docentes en la elaboración de los programas de estudios y en la sistematización del proceso de enseñanza-aprendizaje y que se enfoca a la modalidad de enseñanza (Heredia, 2016).

Basándose en el nuevo modelo educativo, el reto que se busca cumplir es mejorar la calidad de la educación en el país, la cual, durante años, se ha visto afectada tanto por problemas políticos, sociales y económicos, como por la violencia, la pobreza, la lucha entre sindicatos y la diversidad étnica de algunos estados, entre otros. Universidad Veracruzana Virtual (UV2).

La Universidad Veracruzana que tiene como misión apoyar la formación académica de los estudiantes, garantizando que adquieran las habilidades necesarias para el aprendizaje de por vida, promover la distribución social del conocimiento por medio de acciones innovadoras para la formación de recursos humanos, la actualización, reconversión y certificación profesional y el impulso al desarrollo comunitario, mediante el uso intensivo y extensivo de las nuevas tecnologías de la información y la comunicación. (Universidad Veracruzana 2009).

Este nuevo modelo, el cual no se considera un proyecto sexenal, ya que comenzará a finales de la actual administración, tiene muchos retos, específicamente el de la batalla que se libra contra la actual Reforma Educativa. (IES). Organismo o estructura que desempeña labores de docencia, investigación y difusión con el fin de formar profesionistas en las diferentes ramas del conocimiento y preservar, crear y transmitir los bienes de la cultura en relación con el interés social. Las instituciones se rigen por un conjunto de normas, leyes nacionales y reglamentos propios y cuentan, para llevar a cabo su labor, con recursos humanos, materiales, tecnológicos y financieros. (Subsecretaria de Educación Superior 2010) Las instituciones pueden ser públicas o privadas, autónomas, federales o estatales, según el tipo de ingresos de que dispongan. 
Los elementos que intervienen dentro del proceso de enseñanza-aprendizaje son el docente, estudiante, contenidos digitales, foros para poder accesar a este trabajo de investigación de la plataforma Eminus será a través de Red Inalámbrica de la Universidad Veracruzana (RIUV). Servicio de apoyo a las actividades sustantivas de la institución que se ha implementado como una solución para la conexión tanto a la red institucional como a Internet. Ésta fue desarrollada bajo plataforma y aplicaciones de software libre, teniendo gran importancia para la institución debido a su gran crecimiento y expansión, ya que a través de la convergencia de servicios de tercera generación, los alumnos pueden tener acceso a los nuevos sistemas de enseñanza implementados en esta casa de estudios. (Universidad Veracruzana 2008) infraestructura tecnológica.

\section{Definiciones}

Los enfoques teóricos sobre la enseñanza Patterson (1982) decía que no había una teoría de la enseñanza que fuera completamente sistemática y que de hecho no había en las teorías de la enseñanza o de la instrucción tanto desarrollo como lo había en las teorías del aprendizaje. Sin embargo, él se arriesga a presentar las primeras bases para establecer una teoría de la enseñanza. De tal forma que en su libro Bases para una teoría de la enseñanza y psicología de la educación describe el trabajo de cinco investigadores reconocidos para tratar de fundamentar esas bases que él busca proponer.

La educación a distancia es otro tipo de enseñanza donde los estudiantes trabajan desde su casa o desde la oficina, comunicándose con los profesores u otros estudiantes vía e-mail, foros electrónicos, videoconferencia y otras formas de comunicación basada en unidades centrales de procesamiento. (IPN 2010)

E-Learning también llamada enseñanza virtual son situaciones totalmente a distancia desarrolladas a través de las redes telemáticas. Existe una interacción paso a paso a lo largo de los materiales de estudio, así también existe una evaluación momento a momento acerca del impacto de los materiales educacionales sobre los procesos de construcción de conocimientos por parte del alumno.
Tecnologías de Información y Comunicación (TIC) Sistemas tecnológicos mediante los que se recibe, manipula y procesa información, y que facilitan la comunicación entre dos o más interlocutores. Katz, J. Los caminos hacia una sociedad de la información en América Latina y el Caribe. Ed. Naciones Unidas. 2003.

Dato en informática, los datos son representaciones simbólicas (vale decir: numéricas, alfabéticas, algorítmicas, etc.) de un determinado atributo o variable cualitativa o cuantitativa, o sea: la descripción codificada de un hecho empírico, un suceso, una entidad.

Los datos son, así, la información (valores o referentes) que recibe el computador a través de distintos medios, y que es manipulada mediante el procesamiento de los algoritmos de programación. $\mathrm{Su}$ contenido puede ser prácticamente cualquiera: estadísticas, números, descriptores, que por separado no tienen relevancia para los usuarios del sistema, pero que en conjunto pueden ser interpretados para obtener una información completa y específica.

Confidencialidad de datos Resultado del cifrado de datos que evita revelar información a procesos, entidades o individuos no autorizados. Esta información puede ser datos del nivel de aplicaciones o bien parámetros de comunicación. CISCO@ 2010

Base de datos departamental, especializada en el almacenamiento de los datos de un área de negocio específica. Se caracteriza por disponer la estructura óptima de datos para analizar la información al detalle desde todas las perspectivas que afecten a los procesos de dicho departamento. Un datamart puede ser alimentado desde los datos de un datawarehouse, o integrar por sí mismo un compendio de distintas fuentes de información. Sinexxus Business Intelligence 2010

Migración de datos se ve muy bien representada por las siglas ETL, que corresponden a los términos: extracción, transformación y carga. Pese a que se puede aplicar un proceso ETL con otros objetivos, al plantearse qué es la migración de datos es inevitable aludir a su tarea principal: extracción y carga (ya que la transformación no tiene por qué aplicarse en todos los casos, sólo si fuese necesario). 
Hay cuatro opciones principales para llevar a cabo la migración de datos: Combinar los sistemas de las dos empresas o fuentes en uno nuevo. Migrar uno de los sistemas al otro. Mantener la integridad de ambos sistemas, dejándolos intactos, pero creando una visión común a ambos: un almacén de datos. Migrar contenidos educativos a la plataforma virtuales.

Plataforma virtual, es un conjunto de aplicaciones informáticas de tipo síncronas o asíncronas, que facilitan la gestión, desarrollo y distribución de cursos a través de Internet. Este software se instala en el servidor de la Institución que proveerá este servicio a la comunidad. Santoveña (2002) plantea lo siguiente:

Una plataforma virtual flexible será aquella que permita adaptarse a las necesidades de los alumnos y profesores (borrar, ocultar, adaptar las distintas herramientas que ofrece); intuitivo, si su interfaz es familiar y presenta una funcionalidad fácilmente reconocible $\mathrm{y}$, por último, amigable, si es fácil de utilizar y ofrece una navegabilidad clara y homogénea en todas sus páginas". Otro concepto sería: entorno de hardware y software que permite gestionar el desarrollo de actividades formativas de un curso virtual, denominado también "Sistemas de Gestión de Aprendizajes LMS". Existen diversas denominaciones al Término plataforma virtual, como son: Entorno de Aprendizaje Virtual Virtual learning environment (VLE) Sistema de Gestión de Aprendizajes - Learning Management System (LMS) Sistema de Gestión de Cursos - Course Aula Virtual (AV).

Plataforma Eminus sistema de Administración de Ambientes Flexibles de Aprendizaje, desarrollado por la Universidad Veracruzana, el cual permite presentar cursos en línea para distribuirse en Internet o redes internas. Permite la comunicación en forma sincrónica y asincrónica ya que utiliza las tecnologías de la información y la comunicación para aprovechar la facilidad de distribución de materiales formativos $y$ herramientas de comunicación, lo que permite crear un entorno completo para el aprendizaje ayudando a la vez a mejorar los niveles educativos sin límites de tiempo y de distancia, permitiendo a cada estudiante tomar el control de su aprendizaje y formación de una forma independiente y colaborativa. Software Educativo (Universidad Veracruzana 2009)

\section{Análisis de información}

Esta investigación se llevó a cabo en la Región Poza Rica Tuxpan en la Facultad de Trabajo Social fue donde se desarrolló este tema. La Universidad Veracruzana utiliza plataforma Eminus en diferentes Experiencia Educativas y una de ellas es la de Computación básica. En base a esta información se dedujo lo siguiente.

Eminus es Sistema de administración de ambientes flexibles de aprendizaje, que permite presentar y distribuir contenidos educativos, brindando la posibilidad de contar con un "Campus digital" para la comunicación y colaboración sin límite de tiempo y distancia.

La capacitación en el uso del sistema Eminus tiene como objetivo proporcionar los conocimientos necesarios para su óptima utilización así como llevar a cabo sesiones prácticas con el fin de que el usuario conozca de una manera más cercana, la plataforma y aproveche los beneficios que ésta le ofrece para el proceso de enseñanza - aprendizaje. Entrar al portal

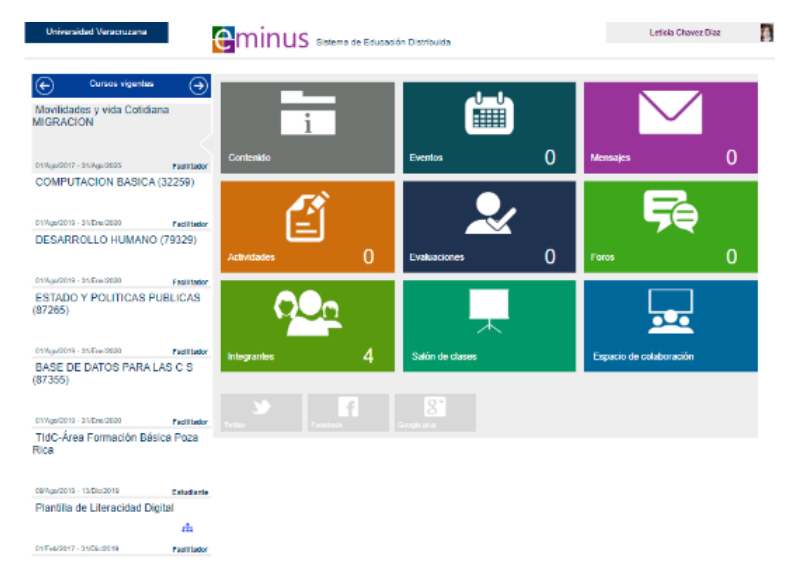

Figura 2 Pantalla de Acceso principal

En esta parte tanto el alumno y el facilitador tienen acceso a los cursos que están inscritos, además de poder acceder a las diferentes herramientas que conforman el curso, tales como evaluaciones, actividades, contenido, etc. 

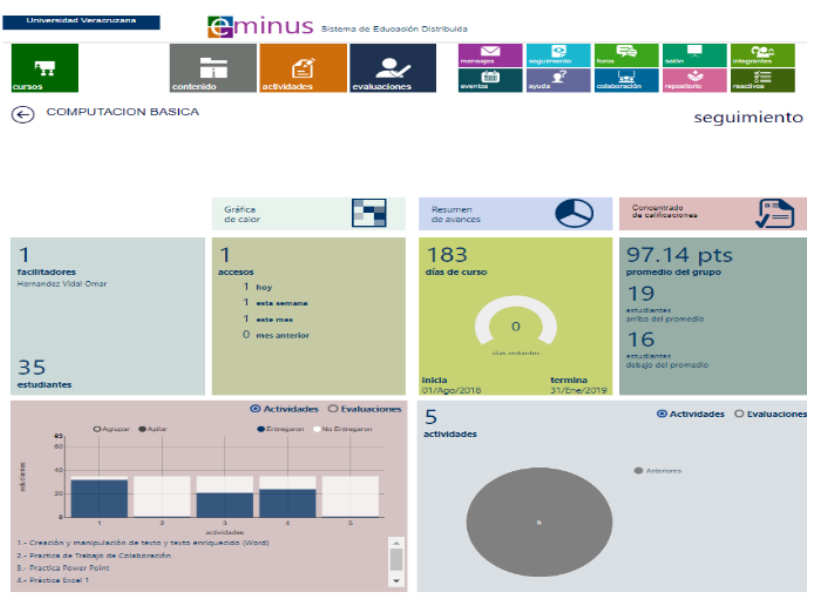

Figura 3 Pantalla principal de Seguimiento

En la siguiente pantalla se muestra el avance general de los alumnos inscritos en el curso, así como el porcentaje grupal de las evaluaciones y actividades, además muestra el promedio del grupo.

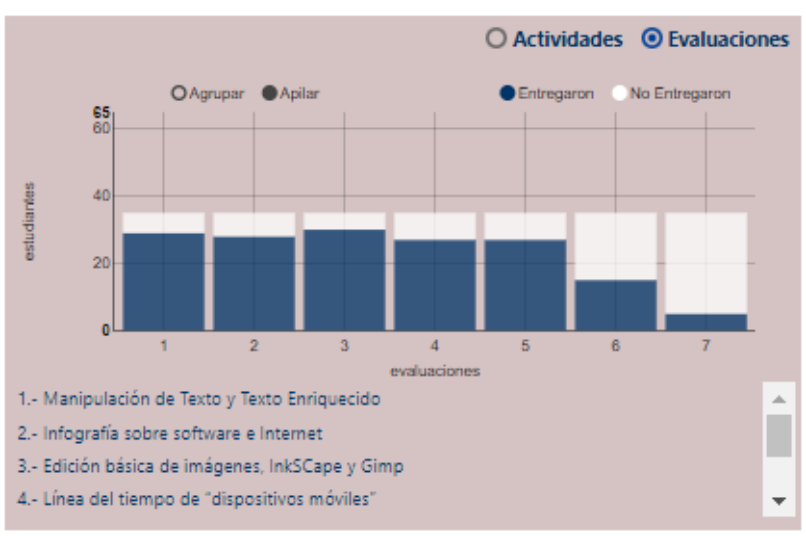

Figura 4 Pantalla de Evaluaciones Fuente: Edición Propia

En esta parte se muestra el porcentaje de los alumnos que entregaron y no entregaron las evaluaciones que conforman el curso registrado en la plataforma Eminus.

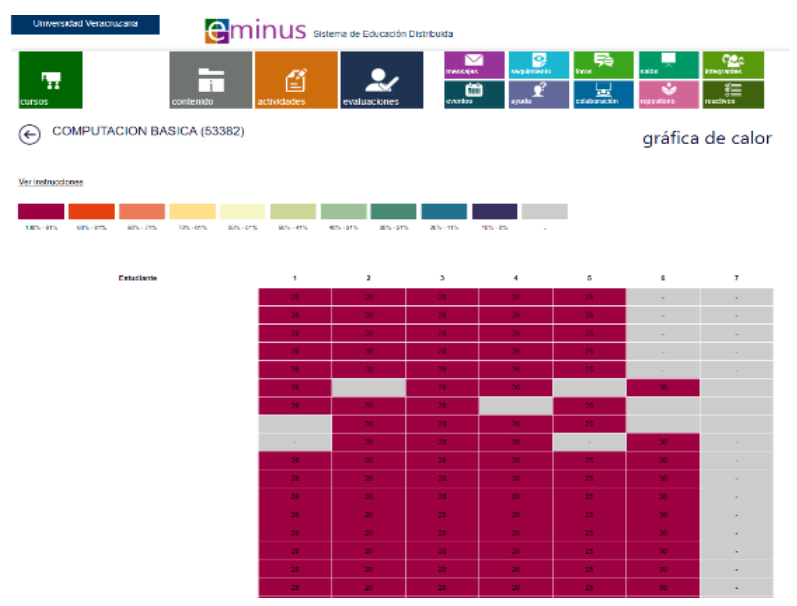

Figura 5 Grafica de Calor

Fuente: Edición Propia
En la siguiente pantalla proporciona información del porcentaje del total de puntos de las evaluaciones, viene en una escala de colores donde color corresponde a un porcentaje.

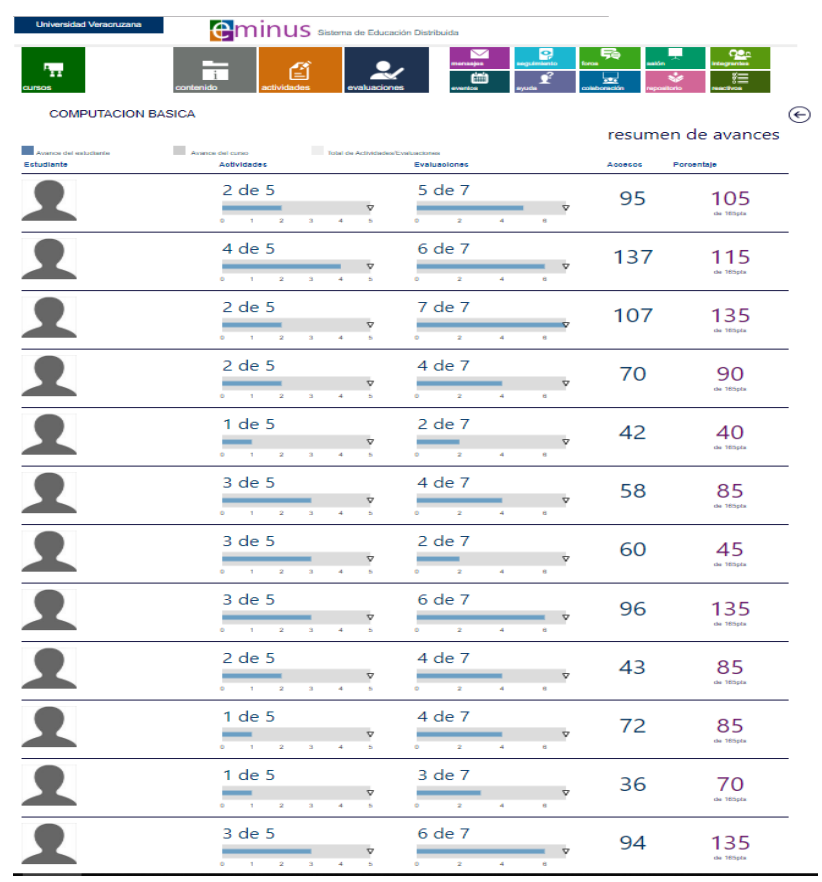

Figura 6 Resumen de avances Fuente: Edición Propia

En esta parte del sistema proporciona información de las actividades, evaluaciones y las puntuaciones finales de los alumnos las cuales son registrados por los docentes.

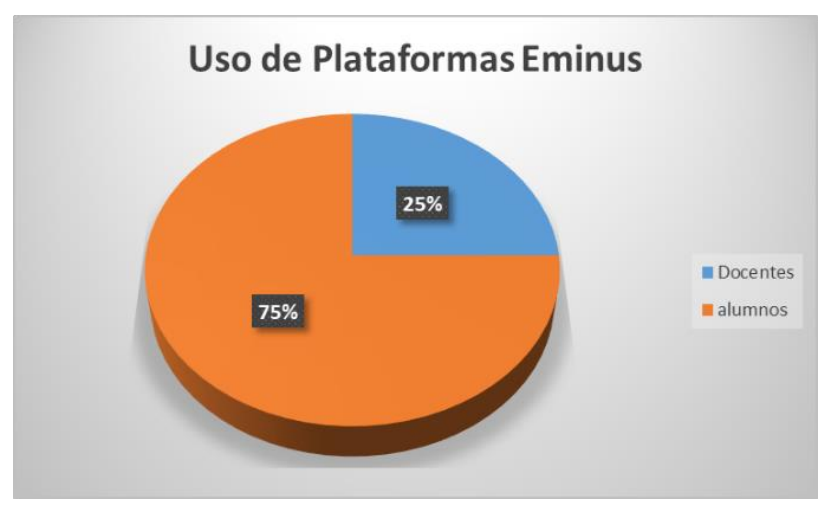

Figura 7 Uso de la Plaforma Eminus Fuente: Edición Propia

En esta grafica se puede mencionar que el uso de la plataforma Eminus solo es del $50 \%$ de los Docentes de la Facultad de Trabajo Social son los que utiliza esta plataforma debido a que están en proceso de migración de datos equivalente a un $25 \%$, cabe mencionar que se presenta mayor índice con 150 alumnos debido a que ellos utilizan las plataformas en tareas actividades y contenidos, representando un $75 \%$. 


\section{Resultados}

Con los resultados obtenidos en esta investigación nos dimos cuenta que la plataforma Eminus realmente es de mucha importancia para los docentes y los estudiantes debido a que son escenarios educativos donde el alumno realiza sus actividades y le permite trabajar en forma colaborativa, sin necesidad de reunirse con sus compañeros o docente, debido a que maneja todo a distancia. Dándole uso a todas las pantallas de acceso principal de la plataforma Eminus y mejorar los resultados de apertura en contenidos y evaluaciones de los programas que los docentes apertura y utilizan para así, mejorar la calidad de la Enseñanza.

\section{Conclusiones}

Esta investigación ha provocado que los docentes y estudiantes cada día incorporen más sus experiencias educativas y tengan acceso en tiempo real y que ellos estipulen su horas para trabajar los contenidos y evaluaciones que los docentes administren desde el portal educativo a la plataforma Eminus, cabe mencionar que también el acceso al seguimiento marca que en las figuras 2,3 y 4 muestra el avance general de los alumnos inscritos en el curso donde el docente puede revisar las actividades y en las figuras 5,6 y 7 puede revisar las tareas y calificar sus respectivas evaluaciones que hizo el alumno. Como recomendación hacia los docentes se pide que exista cursos intensivos para el manejo de la plataforma virtual Eminus para poder mejorar la calidad de E-A de los docentes en forma virtual.

\section{Referencias}

Cabero, A. J. (2007). Nuevas Tecnologías aplicadas a la educación. España: Mc Graw Hill.

Colunga, A. (2005). Eminus. Sistema de educación distribuida. Diapositivas., http://www.cudi.edu.mx/primavera_2005/prese ntaciones/alejandro_colunga.pdf. Mèxico

\section{CISCO}

http://www.fediap.com.ar/administracion/pdfs/ TIC\%20para\%201a\%20Educaci\%C3\%B3n\%20 en\%20Am\%C3\%A9rica\%20Latina\%20-

\%20Guillermo\%20Sunkel\%20-\%20CEPAL.pdf Blog de tencolonia educativa . mayo del 2017 https://edilsmedinawed20.blogspot.com/2017/0 5/que-son-las-plataformas-virtuales-y.html
Fernández March Amparo. (2006). Metodologías activas para la formación de competencias. Educatio sigloXXI, 24, 35-56.

Ferreyra Martínez María Fabiana. (2011). "Implementación y evaluación de un modelo didáctico, basado en enfoques constructivistas, para la enseñanza de Estadística en el nivel superior". 3 de abril de 2016, de Universidad autónoma de baja california Sitio web: http://iide.ens.uabc.mx/blogs/mce/files/2011/03 / TesisMaestria-MFFerreyra.pdf

Galindo, L. (2007). Metodología básica para el desarrollo de sistemas. En A. e. Asociación Mexicana de Metodología de la ciencia y de la Investigación, Memorias del 3er. Congreso Internacional de Metodología de la Ciencia y de la Investigación para la Educación. Campeche, México.

Hernández Sampieri, R., Fernández C., C., \& Baptista, M. (2010). Metodología de la Investigación. México: McGraw Hill

Heredia, B. (25 de mayo de 2016). Modelo educativo ¿qué es y por qué importa? $E l$ financiero. Obtenido de http://www.elfinanciero.com.mx/opinion/model o-educativo-que-es-y-por-que-importa.html

Jiménez, J. (2006). Eminus. Sistema de educación distribuida. Recuperado el 22 de octubre de 2012, de http://www.cudi.mx/primavera_2006/presentaci ones/educación_jcarlos_jimenez.pdf

Manual del estudiante de Plataforma Eminus https://eminus.uv.mx/eminus/manuales/Estudia nte/Manual-Estudiante.pdf

Patterson (1986) Proyecto de vida adolescente, virtual e interactivo, una estrtategia para modificar conductas socialmente indeseables Tamayo y Tamayo, Mario. (1997). El Proceso de la Investigación científica. México: Editorial Limusa S.A

Santoveña Casal Sonia Ma. Metodologia Didactica EN ENTORNOS VIRTUALES DE APRENDIZAJE

https://www.ugr.es/ sevimeco/revistaeticanet/N umero3/Articulos/Formateados/metodologia_di dactica.pdf consultado 2019. 
Universidad Veracruzana. (2018). Eminus. Obtenido de HYPERLINK https://www.uv.mx/dgti/servicios-de-ti/eminus/

Universidad Veracrunaza Eminus Fuente: https://concepto.de/dato-en informática/\#ixzz5z8sGNG3T UV 2009. http://recursostic.educacion.es/observatorio/we b/es/software/software-educativo/1007monografico-el-proceso-de-ensenanzaaprendizaje-mediante-el-uso-de-plataformasvirtuales-en-distintas-etapas-educativas?start $=3$

Universidad Veracruzana. (2017). Manual del facilitador. Eminus. Obtenido de https://eminus.uv.mx/eminus/manuales/Facilita dor/Manual-Facilitador.pdfT. W. Moore. https://blog.powerdata.es/el-valor-de-lagestion-de-datos/bid/397387/Qu-es-la-migracin-de-datos. 